\title{
Migration and Sustainable Development: a critical approach.
}

\author{
Giuseppina Talamo ${ }^{1}$
}

\begin{abstract}
The aim of this paper is to examine international migration studies following an interdisciplinary approach. The starting point is an examination of the increasing international migratory flows, that represents one of the most visible manifestation of the globalization process. Although the recent global crisis has added new concerns in relation to migrant situation particularly in the countries more affected by the recession, analyses of the impact of globalization indicate that migration provides a driving force for sustainable development.. Using recent data, the main characteristics of migration flows in Italy and in Oecd area will be presented. Analyses show that there is a positive correlation between migration and sustainable development, which has often been underestimated or misunderstood.
\end{abstract}

Key Words: Migration, Sustainable Development, Integration.

\section{Introduzione}

Continuano a crescere di giorno in giorno, di ora in ora il numero delle vittime dei naufragi sui barconi della speranza. Uomini, donne, bambini, tutti accomunati da un unico grande sogno: la fuga verso l'Europa. Quell' Europa che annaspa e naufraga nella gestione di questa grande emergenza umanitaria. Quell' Europa che da un lato erge muri, e dall'altro sfida il mare pur di salvare esseri umani da un tragico destino. Scene di barconi pieni di vite umane con le braccia tese a chiedere aiuto in mezzo al quel mare tanto amato, ma anche tanto temuto da quegli esseri umani che vedono il mare come unica via per la fuga e per la speranza di una vita migliore. Di fronte alle scioccanti e raccapriccianti scene dei naufragi, dei tir carichi di morti, delle barriere di filo spinato ed, infine, l'accusa dagli Stati Uniti all'Europa nell'aver fallito nel gestire questa emergenza umanitaria, l'Europa, non riesce ancora a dare una risposta unitaria. Sono stati convocati numerosi vertici straordinari per individuare misure immediate per far fronte a questa emergenza umanitaria e varie sono state le proposte avanzate da ciascun governo: dai muri, ai respingimenti, a politiche di accoglienza, fino alla più recente proposta di fondo fiduciario Ue-Africa del valore di 1,8 miliardi di euro, e il migration compact. L'orientamento dominante sia a livello internazionale che comunitario, sino a questo momento, ha focalizzato il proprio agire con un marcato approccio "funzionalista", ovvero una maggiore attenzione al legame immigrazione-rimesse, immigrazione-fuga di cervelli, con una marginale attenzione alle politiche volte alla promozione del fenomeno 
migratorio e dello sviluppo sostenibile. Alla luce di questa emergenza umanitaria, e a seguito dell'inefficacia delle deboli politiche migratoria si è ritenuto opportuno, soprattutto a livello internazionale, si seguire un approccio che riconsideri il ruolo delle istituzioni nella governance dei flussi migratori: un approccio che riesca a bilanciare aspetti economici, sociali e ambientali per il raggiungimento di uno sviluppo sostenibile. A tal proposito, le Nazioni Unite, per la prima volta, riconoscono nella "2030 Agenda for Sustainable Development", la necessità di facilitare la migrazione a livello globale; la necessità di una migliore governance della migrazion ; la necessità di assicurar e facilitare una migrazione ordinata, sicura, regolare e responsabile e la mobilità delle persone attraverso l'attuazione di politiche migrazione pianificate e ben gestite (UN, 2016).

Obiettivo di questo lavoro è quello di presentare, seguendo un approccio interdisciplinare, la recente letteratura sulle migrazioni evidenziando come la decisione di migrare può essere dovuta non solo a fattori economici e sociali ma anche a cambiamenti ambientali. Quest'ultima prospettiva, anche se ancora poco analizzata, unita agli altri fattori, ci permette ancora di più di capire il modo in cui i fattori di spinta ambientale possono interagire con i fattori economici, sociali, politici e istituzionali. Con l'ausilio di recenti dati verrà presentato il ruolo degli immigrati all'interno del sistema economico e sociale e l'esistenza di una correlazione positiva tra sviluppo sostenibile e migrazioni.

Il lavoro si articola come segue: la sezione 2 discute alcune teorie alla base dei flussi migratori e i risultati dei principali contributi empirici; la sezione 3 presenta un'analisi dei dati sulla presenza degli immigrati nei paesi OCSE. Infine, la Sezione 4 presenta le conclusioni.

\section{Review della letteratura}

I motivi e le cause determinanti le migrazioni internazionali sono molto complesse ed esiste un'ampia letteratura, empirica e teorica, che cerca di analizzare e spiegare questo fenomeno. Lo studio dei flussi migratori, e in particolare, l'attenzione rivolta al fenomeno dell'immigrazione si basa su alcuni dei seguenti fattori: la consistente richiesta di forza lavoro flessibile, le pressioni e i vincoli delle economie dei paesi meno sviluppati da cui provengono gli immigrati, il differenziale reddito pro-capite tra paesi, le guerre civili, le carestie, gli eventi bellici, le reti sociali relazionali-familiari, le politiche migratorie e i sistemi di welfare nei paesi di destinazione.

Recentemente, il dibattito sui flussi migratori internazionali si è anche focalizzato sul nexus (Skelton, 2008), esistente tra immigrazione e sviluppo economico. Diversi studi (teoria dell'integrazione economico-sociale), hanno dimostrato che il fenomeno migratorio può essere visto come uno strumento per promuovere lo sviluppo sia del paese di origine, sia di quello di destinazione. Maggiore attenzione, ad esempio, è stata data all'analisi di aspetti quali: l'apporto contributivo e fiscale degli immigrati nel paese ospitante, le rimesse degli immigrati, la tipologia di lavoro, l'aspetto demografico, le condizioni retributive ed occupazionali. L'impatto che la presenza dei cittadini stranieri ha sul sistema economico dei paesi riceventi, sul mercato del lavoro e sulla sostenibilità del sistema di welfare è oggetto di un acceso dibattito tra attori politici e sociali e si è notevolmente rafforzato a seguito della recente crisi finanziaria. Alcuni autori (Boeri, McCormick e Hanson, 2002; ILO, 2013), suggeriscono come sia importante impostare 
l'analisi del ruolo dell'immigrato nel paese di destinazione secondo l'ottica che considera l'ingresso dei lavoratori immigrati come una fonte di ricchezza per il paese e la loro presenza come un fattore di crescita e sviluppo non solo dal punto di vista economico, ma anche dal punto di vista dell'integrazione socio-culturale. Il contributo del lavoratore immigrato è da molti, infatti, considerato di fondamentale importanza per il mantenimento degli equilibri macroeconomici e di finanza pubblica e per il funzionamento del mercato del lavoro. Alcune ricerche (Boeri, McCormick e Hanson, 2002), evidenziano che paesi con un buoni sistemi di welfare state sono anche i luoghi verso cui tendono gli immigrati. Anche in questo caso, l'impatto dell'immigrazione sul welfare state del paese può essere sia positivo che negativo. Ad esempio, può dipendere dalle caratteristiche socio-economiche degli immigrati rispetto agli autoctoni; l'arrivo degli immigrati può anche avere degli effetti sulla distribuzione dei redditi (Mueller e Tai 2008). A tal proposito la teoria economica si rifà a diversi approcci: l'indicatore dei sistemi di welfare; Borjas (1999), welfare magnet effect, il sistema di welfare può agire come un importante elemento attrattivo dei flussi migratori. Infine, Hatton (2013) afferma che nonostante la recessione, i flussi di immigrazione internazionale non sono mutati considerevolmente e sono stati maggiormente influenzati da modelli di welfare e motivi fiscali piuttosto che dal crescente tasso di disoccupazione.

Altro aspetto da considerare è il ruolo della mobilità all'interno di un sistema globalizzato. Il processo di globalizzazione economica ha acuito il dibattito concernente l'analisi degli effetti dei fattori di spinta ed attrazione, accentuando la contrapposizione tra coloro che vedono il processo di immigrazione come una componente essenziale dello sviluppo economico (approccio classico) e coloro che, invece, ritengono il processo di immigrazione una delle cause principali del crescente impoverimento dei paesi in via di sviluppo (approccio multidisciplinare). Castles (2008), Borjas (1999) affermano che non sussiste una semplice relazione tra povertà ed emigrazione e che, invece, le migrazioni sono contemporaneamente causa e risultato di sviluppo. Lo sviluppo conduce alle migrazioni poiché i miglioramenti economici e educativi rendono le persone capaci di trovare migliori opportunità altrove. Castles, (2009), afferma che le migrazioni sono, anche, il risultato dell'integrazione delle comunità locali e delle economie nazionali nell'ambito delle relazioni globali. L'autore sottolinea come la migrazione è, spesso, il risultato dello sviluppo economico e sociale e a loro volta, le migrazioni, possono contribuire a un ulteriore sviluppo e a migliorare le condizioni economiche e sociali o viceversa possono concorrere alla perpetuazione della stagnazione e della disuguaglianza - A supporto di questa ultima tesi è il fenomeno del brain drain di personale qualificato che migrando impoverisce di risorse il paese di origine. Dal lato del paese di destinazione si assiste, invece, al fenomeno del brain waste: dati recenti confermano che si ha un impiego dei lavoratori immigrati con livello di istruzione medio-alto in mansioni che, invece, richiedono un minor contenuto professionale.

La letteratura economica, proponendo un approccio neoclassico, ha studiato il fenomeno delle migrazioni internazionali come quel processo che nasce da una decisione individuale finalizzata alla massimizzazione del reddito (Todaro, 1969, 1989; Borjas, 1989). Questo approccio afferma che la decisione di ciascun individuo di migrare è collegata ad un'analisi costi-benefici. In generale, un individuo decide di migrare laddove riesce ad essere più produttivo; laddove esistono differenziali nei tassi di salario, nei tassi 
di occupazione, nel reddito pro-capite. Tra le cause delle migrazioni è importante sottolineare, l'importanza dei legami economici e culturali tra i paesi coinvolti nonché il ruolo dei network (o reti sociali) di relazione transnazionali nel foraggiare questo fenomeno.

La teoria del doppio mercato afferma, invece, che le migrazioni originano dalla domanda di lavoro delle moderne società industriali. Nel determinare la scelta di migrare è, così, opportuno distinguere due tipologie di fattori: fattori di spinta e fattori di attrazione. I fattori di spinta caratterizzano i paesi di origine: povertà, diseguaglianze socioeconomiche, caratteristiche demografiche, regimi autoritari, qualità delle istituzioni, carestie, guerre, elevati tassi di disoccupazione. Si parla, invece, di fattori di attrazione quando ci si riferisce ai paesi di destinazione che presentano ai migranti tutta una serie di opportunità sia a livello individuale che di nucleo familiare: libertà civili, elevata qualità della vita, caratteristiche demografiche, maggiori e migliori opportunità di lavoro, salari elevati, politiche migratorie di accoglienza, sistemi di welfare. Quindi, seguendo questo approccio, le cause che spingono alla migrazione sono anzitutto riscontrabili a partire dalle differenze individuate nel mercato del lavoro e quindi agli squilibri tra domanda e offerta. Considerando le differenze tra paesi di origine e paesi di destinazione, la disomogenea ripartizione tra forza lavoro e capitale e un basso equilibrio di mercato salariale, da un lato, e la minor forza lavoro ma più capitale e un mercato salariale più alto, dall'altro, contribuisce a generare una situazione di squilibrio generale che implica spostamenti di lavoratori con bassi salari verso paesi in cui la possibilità di massimizzare il reddito è maggiore. Di conseguenza, si assiste a una diminuzione di offerta di lavoro e un aumento di salario dove il capitale è piuttosto basso e viceversa. Così, i flussi di capitale umano sono la conseguenza delle differenze salariali e vanno a riversarsi sul mercato del lavoro che dovrà essere regolamentato legalmente e correttamente dalle istituzioni.

Un ruolo fondamentale è svolto, anche, dalle rimesse ed è stato dimostrato che esse offrono un contributo massiccio ai conti nazionali dei paese d'emigrazione. La New economics of labour migration (Stark, 1991; Taylor, 1999; Hugo, 1994, 1998) sostiene, invece, che le decisioni di migrare rappresentano una strategia familiare, e non individuale, di massimizzazione del reddito ed opportunità di sopravvivenza (ad esempio, le rimesse). Oltre alla disparità nei livelli di reddito bisogna anche considerare, come causa delle migrazioni, le differenze nei modelli demografici (fertilità, mortalità, crescita forza lavoro e composizione per età).

Infine, Putnam (2007) considera l'importanza della teoria della rilevanza economica del capitale sociale dove le diversità etniche sono un bene valutabile economicamente e il capitale sociale è considerato come un fattore produttivo al pari del capitale fisico e del capitale umano. la World Bank (2014) considera il capitale sociale come un efficiente strumento di politica economica nel ridurre i livelli di povertà ed aumentando l'integrazione sociale.

Altro aspetto che si vuole analizzare in questo lavoro è la relazione tra cambiamenti climatici, catastrofi naturali e progetti "poco" sostenibili da un lato, e processi migratori dall'altro. Anche se a livello giuridico non è riconosciuto alcuno status ai "migranti ambientali", è innegabile come sia necessario considerare il difficile rapporto ambientemigrazioni e sviluppo. Da un lato i "massimalisti” affermano l'esistenza di una relazione 
diretta tra i cambiamenti ambientali e il crescente numero di rifugiati ambientali, dall'altro i "minimalisti" sostengono che sono i fattori sociali, economici e politici che determinano i flussi migrazioni. Tra i fenomeni più controversi di causa ambientale delle migrazioni è annoverato il cambiamento climatico e i suoi effetti, seguono gli incidenti industriali, progetti di sviluppo e strategie di guerra. A livello internazionale e comunitario si cerca di agire attraverso politiche di sviluppo sostenibile volte alla riorganizzazione sociale delle risorse tenendo in considerazione lo stato della tecnologia e le capacità della biosfera di assorbire gli effetti delle attività umane (Rapporto Bruntland).

\section{Alcuni dati}

Secondo i dati OCSE (2014), dagli anni '60 in poi, molti paesi OCSE si sono trasformati da paesi di emigrazione a paesi riceventi con flusso di immigrazione internazionale verso in entrata che ha raggiunto elevati livelli nel 2010 seguendo però un trend non constante: si sono, infatti, alternati dati in crescita nel 2007 seguiti da una leggera diminuzione negli anni 2008-2009, in risposta alla crisi globale, e gli anni successivi, invece, hanno registrato una certa stabilità.

Analizzando i dati relativi ai paesi OCSE di destinazione possiamo giungere a conclusioni diverse per i diversi paesi. Ad esempio la Germania ha registrato un aumento nel flusso in entrata di circa 400.000 persone. Con questi dati nel 2014 la Germania è il secondo più grande paese di destinazione dopo gli USA, accogliendo più del 10\% degli immigrati nell'area OCSE. Una delle principali ragioni è da ricercare nel flusso proveniente dai Paesi dell'Este Europa e dal Sud Europa. Sempre nel 2012 altri paesi OCSE hanno registrato un aumento significativo del flusso di immigrazione: Francia, Svezia e Finlandia. Sempre nello stesso anno, altri paesi EU hanno registrato una forte contrazione in entrata. La Spagna una contrazione del 22\%, Italia del 19\% e Regno Unito dell'11\%. Questi dati confermano che i paesi dell'area OCSE rappresentano poli di attrattività molto differenti per l'eterogeneità delle condizioni economiche, geografiche e sociali. Studi teorici ed empirici hanno dimostrato che la scelta di emigrare non è dettata esclusivamente da considerazioni di tipo economico, ma dipende anche da legali relazionali, dal contesto istituzionale e da fattori culturali e geografici. Dal lato della teoria dell'offerta si parla di fattori push e fattori pull. Dal lato della domanda si analizzano le politiche estere dei paesi di destinazione.

Analizzando i dati relativi alle ragioni che spingono gli individui a migrare, i motivi di ricongiungimento familiare (teoria dei network) si conferma la principale causa. I dati relativi ai ricongiungimenti familiari confermano quanto scritto precedentemente: Svezia tale fenomeno ha avuto un aumento del 26\%; la Svizzera del 16\%; Canada del 15\%; la Francia del 7\%; Spagna, Belgio, Italia e Portogallo una forte riduzione. Mentre per il Giappone e la Korea i dati relativi al flusso di immigrazione rimangono a livelli bassi e stabili, i flussi verso altri paesi OCSE mostrano notevoli e significativi cambiamenti.

In Italia la popolazione residente nazionale è stazionaria, mentre quella straniera è cresciuta ad un tasso medio del 10\% annuo. (Istat, 2013).

La tendenza all'invecchiamento della popolazione italiana è stata frenata dalla crescita rilevante dalla componente immigrata, mediamente molto più giovane di quella italiana. Una prima conclusione che si può trarre è che l'immigrazione rappresenta una fonte di 
incremento demografico per l'Italia, che, invece, presenta un tasso di dipendenza demografica elevato e destinato ad aumentare notevolmente nel tempo (Istat 2013). La rapida crescita del numero immigrati InItalia ha anche determinato un aumento delle imprese individuali gestite da immigrati. Il principale ambito di attività economica degli immigrati in Italia è costituito dal commercio; seguono le costruzioni e le attività manifatturiere. Banca di Italia (2008), analizzando l'incidenza degli immigrati sulla spesa sociale, ha stimato che gli immigrati ricevono il 2,5\% di tutte le spese di istruzione, pensione, sanità e prestazioni di sostegno al reddito, e cioè, all'incirca la metà di quello che assicurano in termini di gettito.

La conclusione che da queste percentuali si può trarre è che gli immigrati hanno finora soddisfatto una domanda di lavoro proveniente dal sistema produttivo italiano che riguarda lavori a bassa qualifica e mansioni di assistenza. Lavori, questi ultimi, che sono stati, infatti, al centro dei provvedimenti di regolarizzazione recentemente adottati nel nostro paese. L'inserimento dei lavoratori immigrati nel mercato del lavoro italiano è caratterizzato da un processo di "etnicizzazione" (ILO, 2013). Il settore terziario, in particolare i lavori di cura e di assistenza alle persone, la professione infermieristica e le attività alberghiere e della ristorazione, assorbe gran parte della forza lavoro straniera.

I lavoratori stranieri occupati partecipano, inoltre, alle entrate nelle casse dello stato tramite i contributi versati a carico del lavoratore e quelli a carico dell'impresa. Secondo le ultime stime disponibili di Unioncamere (2013) i cittadini stranieri presenti in Italia hanno contribuito per il 12,8\% alla creazione del valore aggiunto, corrispondente a un dato assoluto pari a 178,5 miliardi di euro. Sebbene, negli ultimi anni, a causa della crisi, vi sia stata una contrazione del tasso di occupazione anche per i cittadini stranieri, ciò non sembra aver inciso sul loro contributo alla creazione di valore aggiunto che mostra tra il 2005 e il 2011 un incremento costante.

\section{Conclusioni}

La crescita e l'evoluzione del fenomeno delle migrazioni internazionali richiede che tale fenomeno sia accompagnato da obiettivi politici e sociali condivisi e non azioni sporadiche e a hoc dettate da eventi eclatanti senza una prospettiva a lungo termine. Ciò dovrebbe aiutare i governi a capire meglio le politiche da adottare, i sistemi di welfare da seguire, il mercato del lavoro per poter riorientare le politiche occupazionali e migratorie a beneficio di tutti i lavoratori, e non solo dei migranti. L'aumento delle immigrazioni irregolari e la tratta degli esseri umani rappresentano, infatti, importanti problemi che la comunità internazionale deve essere in grado di affrontare in termini di protezione dei diritti umani e del lavoro. Forse bisognerebbe trattare tu ciò non come una emergenza, ma come il risultato di una trasformazione sociale e di sviluppo a livello globale. Oltre alla necessità di un accordo comune per contrastare questa emergenza umanitaria e la tratta di vite umane, sarebbe opportuno che si iniziasse a riflettere su due concetti: accoglienza ed integrazione. Accogliere come mettere insieme e integrazione come stare insieme ed essere parte attiva della società. Si porrebbe così fine al continuo etichettare gli esseri umani come profughi, richiedenti asilo, clandestini, migranti ed immigrati: tutte etichette che alimentano una guerra tra esseri umani in fuga e che risvegliano in alcuni pensieri xenofobi. Si tratta di essere umani, di popoli, che fuggono dalla guerra, dalla 
fame, dalle malattie, dalle persecuzioni, dalla mancanza di cibo e lavoro. Le misure immediate devono prevedere un contrasto serio e significativo alla tratta di esseri umani, levando il privilegio alle organizzazioni criminali di gestire la paura e i sogni di uomini, donne, bambini in fuga da guerre e non solo. Un recente rapporto OCSE (2015), sottolinea come il processo di integrazione degli immigrati è uno dei principali obiettivi nell'agenda dei policy makers di diversi paesi europei e dell'area OCSE. Dal rapporto emerge che quando gli immigrati si integrano con successo, essi contribuiscono positivamente al sistema economico - sociale del paese ospitante. Infine, il processo di integrazione non può essere immediato e ha bisogno di tempo, opportunità, solidarietà ed accoglienza verso tutti.

Infine, anche l'agenda di sviluppo dell'ONU (2014) offre l'occasione di promuovere una maggiore integrazione dell'individuo immigrato nel paese di destinazione, promuovendo un lavoro dignitoso e una maggiore protezione sociale.

Un'ipotesi che a livello europeo si sta valutando è quella di replicare il modello del Fondo europeo degli investimenti strategici (Efsi), ovvero un fondo che si basa su garanzie comunitarie, Banca europea per gli investimenti e una leva finanziaria proveniente dagli investitori. Altro piano strategico di intervento per lo sviluppo delle aree del Nord Africa, il Sahel e il Corno d'Africa, è il migration compact.

Bisogna inquadrare la situazione nell'ottica di una questione politica e di sicurezza internazionale che coinvolge non solo l'Europa ma tutto l'Occidente. L'attuale situazione che l'Occidente sta vivendo, non è solo la conseguenza di guerre e povertà, ma è, anche, la cattiva redistribuzione delle risorse, delle ricchezze a livello globale. Siamo di fronte a una crisi di lungo periodo.

Di conseguenza, rafforzare i controlli alle frontiere e proporre solo aiuti allo sviluppo non possono rappresentare un ostacolo all'immigrazione. Nel primo caso si rischierebbe un affinamento e rafforzamento della rete criminale transnazionale che pilota l'immigrazione clandestina verso l'Europa. Nel caso del canale ufficiale dell'aiuto allo sviluppo, si rischia di limitare ancor di più la libertà di movimento del migrante e non sia nessuna evidenza empirica che lo sviluppo nel paese di origine possa eliminare l'immigrazione.

\section{Bibliografia}

ATLANTE DELL'IMMIGRAZIONE IN ITALIA (2011), Carocci Editore.

B., HOLLIFIELD J. F. (2007), Migration Theory: Talking across Disciplines, Routledge.

Boeri T., Hanson G. and B. McCormick, (2002), Immigration Policy and the Welfare System, Oxford University Press.

Boeri T. (2010), Immigražione non è uguale a criminalità, lavoce.info.

Borjas, G. J. (1987), "Self-Selection and the Earnings of Immigrants", American Economic Review, Vol. 77 (4), pp. 531-553

Borjas G. J (1994), The economics of immigration, Journal of Economics Literature 32, 1667-1717.

Borjas G. J. (1999), The Economic Analysis of Immigration, in O. C. Ashenfelter and D. Card (Eds.),

Borjas G. J (1995), "The economic benefits if immigration of immigration", Journal of Economic Perspectives 9, 3-22.

BUCKLEY, F.H. (1996), The Political Economy of Immigration Policies, International Review of Law and Economics; 16(1), 81-99.

BOYD, (1989), Family and Personal Networks in International Migrations: Recent Developments and New Agendas, in International Migration Review, Vol.23, N. 3, Pg. 638 - 670. 
CARITAS/MIGRANTES (2011), Dossier Statistico Immigrażone:Oltre la crisi, insieme Idos, Roma.

CARITAS/MIGRANTES (2010), Dossier Statistico Immigrazione:Oltre la crisi, insieme Idos, Roma.

CARITAS/MIGRANTES (2009), Dossier Statistico Immigraz̧ione: Immigrazione, Conoscenze e Solidarietà, Idos, Roma.

Castels S. (2000), Ethnicity and Glonalization: from Migrant Worker to Transnational Citizen, London: Sage, 2000.

Castels s, S. -Miller, M.J. (2003), The Age of Migration.International Population Movements in the Modern World, Third Edition, New York - London, The Guilford Press.

ILO, (2013), Global Employment Trends, 2013

ISTAT (2013), Rapporto ISTAT sull'immigrazione.

OECD (2014), Migration Policy Debate, may 2014.

ONU (2014), Millennium Development Report,UN 2014.

PORTES A. (1995), Economic Sociology of Immigration, New York: Russell Sage Foundation

Putman R. D. (2007), E Pluribus Unum: Diversity and community in the twenty-first century. The 2006 Johan Skytte prize lecture. Scandinavian Political Studies, 30(2), 137-174

Skelton R. (2008), International Migration as a tool in development policy: a passing phase?, in Population and Development Review, 34 (1): 1-18.

STARK, 0. (1991), The Migration of Labour. Cambridge: Basil Blackwell

TAYLOR J. E. (1999), The New Economics of Labour Migration and the Role of Remittances un the Migration Process, International Migration, Volume 37, Issue 1, Pages 63 - 68, March 1999.

TAYLOR J. E. (2006), International Migration and Economic Development, UN.

Todaro, M. (1969), A Model of Labor Migration and Urban Unemployment in Less Developed Countries, The American Economic Review 59 (March 1969): 138-148.

UNIONCAMERE - MINISTERO del LAVORO (2013), Terzo Rapporto Annuale: Gli Immigrati nel Mercato del Lavoro in Italia, 2013.

UN 2030 Agenda for Sustainable Development, http://www.un.org/sustainabledevelopment/developmentagenda/

World Bank (2014), World Development Report, 2014. 